\title{
O Bolsa Família Aumentou a Eficiência para a Segurança Alimentar?
}

Has the Bolsa Familia Increased Efficiency for Food Security?

\section{Abstract}

A alimentação adequada é direito fundamental do ser humano, inerente à dignidade da pessoa humana e indispensável à realização dos direitos consagrados na constituição federal, devendo o poder público adotar as políticas e ações que se façam necessárias para promover e garantir a segurança alimentar e nutricional da população. Dentre as muitas políticas brasileiras elaboradas para combater a fome desataca-se o Bolsa Família, implantado em 2003. Na literatura há indícios de que uma das causas da queda da insegurança alimentar foi o aumento da renda, onde os programas de transferência de renda como o Bolsa Família foram muito importantes. O objetivo desse artigo é determinar a eficiência relativa do Bolsa Família nas unidades federativas para a segurança alimentar. $\bigcirc$ método aplicado foi a Análise Envoltória de Dados (DEA) - Modelo Slack-Based Measure (SBM) assumindo Retornos Variáveis de Escala (VRS) para os anos de 2004, 2009 e 2013, em conjunto com análise de janela, e o método de desempate Índice Composto de Leta et al. (2005). Assim, os resultados após desempate mostraram que o estado onde o Bolsa Família foi considerado eficiente no período foi Santa Catarina.
Adequate food is a fundamental right of the human being, inherent in the dignity of the humans and indispensable for the realization of the rights established in the federal constitution, and the public authority must adopt the policies and actions that are necessary to promote and guarantee the food and nutritional security of the population. Among many Brazilian policies designed to combat hunger, the Bolsa Familia program was implemented in 2003. Evidence is found in the literature supporting that one of the causes of the fall in food insecurity has been the increase in income, where income transfer programs such as Bolsa Familia were very important. The aim of this paper is to determine the relative efficiency of the Bolsa Familia in the federative units for food security. The applied method was the Data Envelopment Analysis (DEA) - Slack-Based Measure (SBM) with variable returns to scale (VRS) assumption for the years of 2004, 2009 and 2013, together with window analysis, and the Composite Index tie-breaking method from Leta et al. (2005). Thus, the results after the tie-breaking showed that the state where Bolsa Familia was considered most efficient in the analyzed period was Santa Catarina. 


\section{INTRODUÇÃO}

A alimentação adequada é direito fundamental do ser humano, inerente à dignidade da pessoa humana e indispensável à realização dos direitos consagrados na Constituição Federal, devendo o poder público adotar as políticas e ações que se façam necessárias para promover e garantir a segurança alimentar e nutricional da população (BRASIL, 2006).

Ferreira Vinhas (2010) afirma que, a segurança alimentar é definida como o acesso da população a alimentos. A carência nutricional é a principal causa da desnutrição e até mesmo da fome, que é considerada como a pior consequência da insegurança alimentar, porque impede a consolidação do conceito de soberania alimentar do país.

Anand e Harris (1990) corroborando com Ferreira Vinhas (2010) afirmam que, a dimensão da fome e da insegurança alimentar pode ser considerada como um importante indicador da qualidade de vida de um país.

Neste contexto, o Brasil foi reconhecido como a sétima economia mundial em 2016 (FMI, 2016). Apesar do êxito econômico, o país é desenhado por profundas heterogeneidades sociais entre suas regiões e seus habitantes, o que inclui a segurança alimentar.

Em razão disso, o país tem planificado diversas políticas para combate à pobreza e fome, que impactaram diretamente nas problemáticas desencadeadas pela insegurança alimentar. Um dos mais importantes programas estruturados foi o Programa Bolsa Família (PBF), promulgado pela Lei Federal $\mathrm{n}^{\mathrm{o}} 10.836$, cuja finalidade é melhorar as condições socioeconômicas das famílias pobres e extremamente pobres por meio de transferência direta de renda (BRASIL, 2004a; BRASIL, 2017a).

De acordo com a Pesquisa Nacional de Amostras por Domicílio (PNAD), o percentual de brasileiros vivendo em situação de insegurança alimentar, em 2004, era de 39,1\% e caiu para 25,8\% em 2013. O cenário é ainda mais preocupante em crianças de 0 a 4 anos, sendo a proporção de insegurança alimentar nessa população de 34,2\%, em 2013 (PNAD, 2017a; PNAD, 2017b).

Associado a essa conjuntura, as desigualdades regionais são muito expressivas. Por exemplo, no ano de 2103 o percentual de pessoas em situação de insegurança alimentar no Maranhão representava 60,86\%. Em contrapartida, os estados do Espírito, Santo Santa Catarina e São Paulo, apresentaram percentual inferior a $12 \%$ (PNAD, 2017b).

De acordo com Hoffmann (2014), o país apresentou uma trajetória de queda na insegurança alimentar. Os fatores fundamentais para a segurança alimentar são: a renda domiciliar per capita, grau de escolaridade, disponibilidade de água encanada, luz elétrica e esgoto apropriado e a estabilidade da renda. Nesse sentido, destaca-se a importância das transferências de renda (Bolsa Família, Benefício da Prestação Continuada, aposentadorias rurais, etc.) e o crescimento do valor real do salário mínimo.

Conforme o exposto, este artigo tem como objetivo determinar a eficiência relativa das Unidades Federativas brasileiras em converter os investimentos no Bolsa Família e PIB per capita em melhorias nos indicadores de segurança alimentar, através da Análise Envoltória de Dados (DEA). Para tanto, 
torna-se imperativo identificar o modelo de relação entre gastos do governo com o PBF e o impacto para melhorar os indicadores nutricionais e acesso da população à alimentação.

O restante do artigo está estruturado da seguinte forma: a seção 2 apresentará uma breve revisão acerca do contexto do PBF e (in) segurança alimentar no Brasil; a seção 3 descreverá a base de dados e o ferramental metodológico aplicado para o cálculo de eficiência; a seção 4 discorrerá sobre os resultados e discussões; e; por fim, a seção 6 apresentará as considerações finais.

\section{REVISÃO DE LITERATURA}

Para o presente trabalho, procurou-se por meio de uma revisão de literatura, abordar conteúdo relacionado aos efeitos do Programa Bolsa Família (PBF) para os indicadores de segurança alimentar.

O Programa Bolsa Família destacou-se no interior do Nordeste, sendo o programa de maior eficácia no aspecto de transferência de renda, pois dinamizou a economia dessas localidades por meio do aumento do poder de consumo da população (FERREIRA VINHAS, 2010).

Ramos e Cuervo (2012) estudaram o Bolsa Família sob o aspecto do direito à alimentação, pois além de transferir renda, o programa visa a garantia de direitos sociais básicos. Os autores ainda destacaram a importância das estruturações políticas em conjunto ao PBF para o desenvolvimento da segurança alimentar e nutricional e do direito humano à alimentação adequada.

Cotta e Machado (2013) procuraram avaliar na literatura disponível os efeitos do Programa Bolsa Família para a promoção da segurança alimentar e nutricional no Brasil. Concluiu-se que, a garantia do direito a segurança alimentar exige programas complementares para enfrentar a desnutrição, o sobrepeso e a obesidade. Sendo assim, o programa poderia ser mais exitoso se fosse combinado com outros tipos de políticas públicas voltadas para a promoção da alimentação adequada.

Custódio et al. (2013) investigaram a evolução da distribuição dos recursos da União para a Política Nacional de Segurança Alimentar e Nutricional (PNSAN), no período de 2004 a 2010. Os autores constataram que houve crescimento dos recursos alocados para os programas da PNSAN (US\$ 15 bilhões em 2010, representando um aumento de 82\% em relação a 2004), sendo a maior proporção dos recursos destinada ao PBF. Assim, concluiu-se que, o montante de recursos para a PNSAN vem crescendo, estimulando a segurança alimentar e nutricional.

Carvalho et al. (2013) estudaram métodos para avaliar programas e políticas públicas como o Programa Nacional de Suplementação de vitamina A e o PBF. Com relação ao PBF, o estudo possibilitou conhecer a percepção dos vários atores sociais envolvidos, além da necessidade de melhorar aspectos educacionais e de saúde para promover a autonomia dos beneficiários em relação ao autocuidado e corresponsabilização no monitoramento das ações do programa.

Martins et al. (2013) realizaram uma revisão sistemática de literatura para analisar a influência do Programa Bolsa Família em minimizar os entraves relativos à alimentação e nutrição. Verificou-se que todas as pesquisas indicaram maior consumo de alimentos entre os beneficiários. 
Wolf e Barros Filho (2014) procuraram estudar os impactos do PBF nas condições de saúde e nutrição da população. Por meio de uma revisão sistemática de literatura, os autores ressaltaram a dificuldade em encontrar-se dados sobre os aspectos nutricionais do PBF e concluíram que o programa não está alterando efetivamente o estado nutricional dos beneficiários, conforme seus objetivos iniciais.

Nessa conjuntura, é possível observar uma riqueza de conteúdo que engloba o Programa Bolsa Famílias com aspectos voltados a segurança alimentar. Contudo, não foram encontrados estudos avaliando a segurança alimentar por meio da Análise Envoltória de Dados, conforme proposto nessa pesquisa. O próximo tópico discorre sobre o método utilizado no presente estudo.

\section{PROCEDIMENTOS METODOLÓGICOS}

Nesta seção, serão apresentadas a base de dados, a seleção de variáveis para o modelo de eficiência, além do ferramental econométrico e da Análise Envoltória de Dados (DEA).

\subsection{Base de Dados}

De acordo com o Ministério Público Federal (MPF), a Escala Brasileira de Insegurança Alimen$\operatorname{tar}$ (EBIA), classifica o acesso a alimentos em quatro escalas: regular e permanente, leve, moderada e grave (BRASIL, 2004b). Essa metodologia foi aplicada na Pesquisa Suplementar de Segurança Alimentar 2004, 2009 e 2013, realizada pela PNAD, cujos os dados serão utilizados para o presente artigo (PNAD, 2017a; PNAD, 2017b).

Bickel et al. (2000) salientaram que a EBIA foi baseada na escala de Departamento de Agricultura dos Estados Unidos. Segundo Radimer et al. (1992) essa escala foi desenvolvida pela Universidade de Cornell, por meio de métodos qualitativos a fim de avaliar a insegurança alimentar e a fome entre mulheres em situação de vulnerabilidade

A base de dados das transferências de recursos do Bolsa Família foi retirada do Portal da Transparência dos Recursos Federais, vinculado ao Ministério da Transparência, Fiscalização e Controladoria- Geral da União (BRASIL, 2017b). Os dados de mortalidade e desnutrição infantil foram obtidos pelo Departamento de Informática do Sistema Único de Saúde (DATASUS, 2017A; DATASUS, 2017b). A proporção de pobres, de extremamente pobres e expectativa de vida foram retiradas do Instituto de Pesquisa em Economia Aplicada - IPEA. (IPEA, 2017a; IPEA 2017b e IPEA 2017c).

\subsection{Seleção de variáveis e aplicação no modelo}

Com base na seleção de dados disponíveis em combinação a literatura consultada, as variáveis selecionadas para o presente estudo podem ser observadas na Figura 1:

Figura 1 - Variáveis selecionadas 

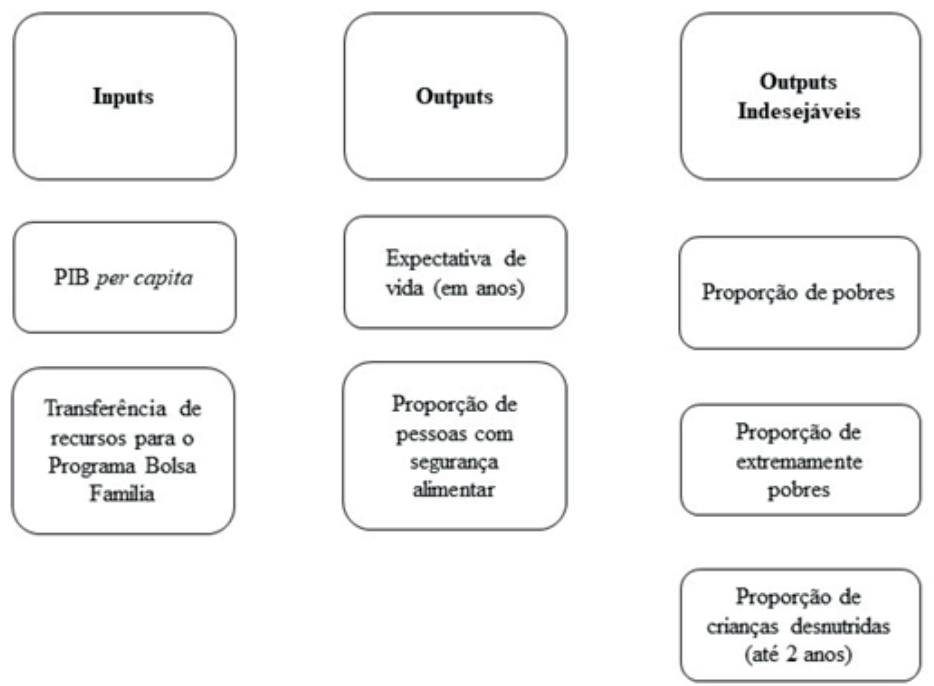

Taxa de mortalidade
infantil
(por mil nascidos)

Fonte: Elaboração dos autores.

Os inputs selecionados para o modelo de eficiência foram as transferências de recursos para o Programa Bolsa Família (BF) e o PIB per capita (Y). Os outputs foram o percentual de pessoas em situação de segurança alimentar (SA) e a expectativa de vida (EV). Também foram considerados como outputs indesejáveis (que são tratados de forma inversa) o percentual de pobres (P), o percentual de extremamente pobres (EP), o percentual de desnutrição infantil de crianças até 2 anos (DI) e a taxa de mortalidade infantil (MI). Os outputs indesejáveis foram tratados de forma inversa como 1/output.

\subsection{Análise econométrica}

Para validação das variáveis utilizou-se a análise econométrica aplicando uma análise de regressão linear múltipla para validar a relação de inputs e outputs (Expressão 1).

$$
\log \gamma \text { outputs }=\beta 0+\beta \text { input } 1+\beta \text { input } 2+\varepsilon
$$

Em que: $\log \gamma$ é uma variável dependente; $\beta 0$ é o intercepto; $\beta$ input 1 são as transferências de recursos para o Programa Bolsa Família (BF); $\beta$ input2 é o PIB per capita (Y) $\varepsilon$ é o erro aleatório.

De acordo com Greene (2003) a regressão log-log permite interpretar os parâmetros como elasticidades. Para corrigir os problemas de heteroscedasticidade, autocorrelação e endogeneidade, que podem ocorrer quando se trabalha com dados em painel ou série de dados no tempo, optou-se pelo Método de Mínimos Quadrados Generalizados Factíveis (MQGF). 


\subsection{Análise de eficiência}

Umas destas técnicas amplamente utilizada para mensurar a eficiência é a Análise Envoltória de Dados (DEA) (VILELA, 2004). A DEA avalia a eficiência relativa de Unidades tomadoras de decisão (Decision Making Units - DMU), responsáveis pela transformação de entradas (inputs) em saídas (outputs). Uma das premissas das DMUs é a homogeneidade em suas funções para ser possível comparar seus desempenhos relativos.

Em seguida é gerado um ranking que relaciona cada DMU a um valor quantificador de sua eficiência relativa. São eficientes aquelas que apresentarem a mínima proporção a que podem reduzir os consumos de inputs sem diminuir a quantidade produzida de outputs (COELLI et al., 2005).

Baseada em estudos prévios de Farrell (1957), a DEA foi apresentada pela primeira vez por Charnes, Cooper e Rhodes. (1978) com o modelo de Retornos Constantes de Escala (CRS). Para medir os ganhos de escala em eficiência, Banker, Charnes e Cooper (1984) desenvolveram o modelo de Retornos Variáveis de Escala (VRS).

Além disso, de acordo com Mariano e Rebelatto (2014), a DEA pode se diferenciar de acordo com os modelos empregados, o tipo de retorno de escala (crescente, constante ou decrescente), orientação (ao input ou output) e pela própria combinação de variáveis (inputs e outputs)

Para a presente pesquisa, optou-se pelo modelo Slack-Based Measure (SBM), assumindo Retornos Variáveis de Escala (VRS), orientado ao output, ou seja, é levada em consideração a escala da DMU (Unidade Federativa). A orientação do modelo se justifica pela atual conjuntura brasileira, em que se espera que os gastos públicos no Programa Bolsa Família se mantenham constantes e que os indicadores relacionados a segurança alimentar (outputs) sejam maximizados.

A seguir pode-se observar a formulação do modelo SBM proposto por Tone (2001) (Expressão 2 a 6):

$$
\operatorname{Min} \tau=t-\left(\frac{1}{m}\right) \sum_{i=1}^{m} \frac{s_{i}^{-}}{x_{i 0}}
$$

S.a.:

$$
\begin{array}{ll}
\left(\frac{1}{n}\right) \sum_{j=1}^{n} \frac{s_{j}^{+}}{y_{j 0}}+t=1 & \\
\sum_{k=1}^{z} \Lambda_{k} x_{i k}+S_{i}^{-}-\mathrm{tx}_{\mathrm{i} 0}=0 & \mathrm{i}=1,2, \ldots, \mathrm{m} \\
\sum_{k=1}^{z} \Lambda_{k} y_{j k}-S_{j}^{+}-\mathrm{ty}_{\mathrm{j} 0}=0 & \mathrm{j}=1,2, \ldots, \mathrm{n} \\
\Lambda_{k} \geq 0, S_{i}^{-} \geq 0, S_{j}^{+} \geq 0 \text { and } \mathrm{t}>0 &
\end{array}
$$


Onde: $\tau$ é a eficiência; $\mathrm{S}_{\mathrm{i}}^{-}$é a folga do input; $\mathrm{S}_{\mathrm{j}}^{+}$é a folga do output; $\Lambda_{\mathrm{k}}$ é a contribuição da DMU k para a DMU analisada; $t$ é o fator de linearização do modelo; $\mathrm{x}_{\mathrm{i} 0}$ é o input da DMU analisada; $\mathrm{x}_{\mathrm{ik}}$ é o input da DMU k; $\mathrm{y}_{\mathrm{j} 0}$ é o output da DMU analisada; $\mathrm{y}_{\mathrm{jk}}$ é o output da DMU k; m é o número de inputs; $s$ é o número de outputs; e z é o número de DMUs.

Além disso, para considerar a esfera temporal, aplicou-se a Análise de Janela (window analysis). Por meio dessa técnica, é possível observar a evolução da eficiência, pois considera cada DMU da série temporal como uma unidade distinta (CAMIOTO, 2014). Essa análise separa os períodos em janelas, ou seja, em diferentes grupos de dados.

\subsection{Método de desempate por Índice Composto/DEA}

Para desempatar as DMUs consideradas eficientes, foi utilizado o método de desempate, chamado de Índice Composto, que pode ser calculado da forma proposta por Leta et al. (2005) (Expressão 7):

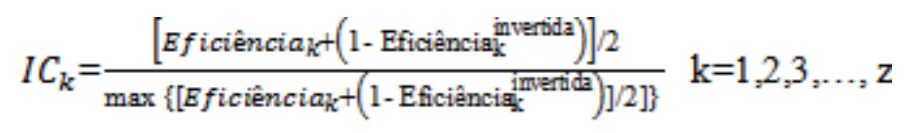

Onde, Eficiência ${ }_{\mathrm{k}}$ é a eficiência da DMU k; Eficiência ${ }_{\mathrm{k}}{ }^{\text {invertida }}$ é a eficiência invetrtida da DMU k; $I C_{\mathrm{k}}$ é o índice composto das eficiências normalizado, e z é o número de DMUs.

Esse método consiste basicamente em calcular a média ponderada e normalizada (pelo valor máximo) das eficiências padrão e invertida.

\section{RESULTADOS E DISCUSSÕES}

Foi avaliada a eficiência relativa das Unidades Federativas brasileiras em converter os investimentos no Bolsa Família e PIB per capita em melhorias nos indicadores de segurança alimentar. A matriz de correlação e as regressões lineares foram mensuradas pelo software STATA. Os resultados da análise econométrica são sintetizados na Tabela 1 .

Tabela 1 - Regressão: coeficientes e valor-p do modelo de eficiência. 


\begin{tabular}{|c|c|c|c|}
\hline & & \multicolumn{2}{|c|}{$\begin{array}{c}\text { Variáveis } \\
\text { independentes }\end{array}$} \\
\hline \multicolumn{2}{|c|}{$\begin{array}{c}\text { Variáveis } \\
\text { dependentes }\end{array}$} & $B F$ & $Y$ \\
\hline \multirow{2}{*}{$\mathbf{P}$} & Coef. & $-0,00468$ & $-0,918 * * *$ \\
\hline & Valor p & $(0,0375)$ & $(0,0642)$ \\
\hline \multirow{2}{*}{ EP } & Coef. & 0,0429 & $-1,137 * * *$ \\
\hline & Valor p & $(0,0286)$ & $(0,0508)$ \\
\hline \multirow{2}{*}{ SA } & Coef. & $-0,0176 * *$ & $0,306^{* * * *}$ \\
\hline & Valor $\mathbf{p}$ & $(0,00829)$ & $(0,0163)$ \\
\hline \multirow{2}{*}{ DI } & Coef. & $-0,0932 * * *$ & $-1,552 * * *$ \\
\hline & Valor $\mathbf{p}$ & $(0,0225)$ & $(0,0434)$ \\
\hline \multirow{2}{*}{ MI } & Coef. & 0,00960 & $-0,445 * * *$ \\
\hline & Valor p & $(0,0132)$ & $(0,0251)$ \\
\hline \multirow{2}{*}{ EV } & Coef. & $0,00309 * * *$ & $3,818 * * *$ \\
\hline & Valor p & $(0,000712)$ & $(0,00912)$ \\
\hline
\end{tabular}

Fonte: Elaborado pelos autores

A análise de regressão demonstrou que o Bolsa Família apresentou significância estatística para a queda desnutrição e mortalidade infantil, e para o aumento da expectativa de vida. Além disso, o programa pode ter contribuído para o decréscimo da percentagem de pessoas em situação de insegurança alimentar, o que vai ao encontro com os estudos de Ferreira Vinhas (2010), Ramos e Cuervo (2012), Cotta e Machado (2013) e Wolf e Barros Filhos (2014).

O PIB per capita apresentou ser significante para a queda de pobres e extremamente pobres, da desnutrição e da mortalidade infantil, e contribuiu para o aumento da expectativa de vida, ou seja, colaborou para a melhora desses indicadores.

Sendo assim, baseado na validação econométrica, os inputs utilizados foram as transferências destinadas ao Bolsa Família (BF) e o PIB per capita (Y) e os outputs foram a percentagem de pobres $(\mathrm{P})$, percentagem de extremamente pobres (EP), a percentagem de pessoas em situação de segurança alimentar (SA), a percentagem de desnutrição infantil (DI), a taxa de mortalidade infantil (MI) e a expectativa de vida $(\mathrm{EV})$.

A Tabela 2 apresenta os resultados dos escores de eficiência do modelo proposto. Pode-se observar que das 27 DMUs analisadas, 16 são consideradas eficientes em todos os períodos, até mesma utilizando aspectos temporais da Análise de Janela. Apenas o Rio de Janeiro e o Amazonas são ineficientes em todos os períodos. Já que mais da metade das DMUs estão empatadas, aplicou-se o método de desempate do Índice Composto de Leta et al. (2005) descrito no tópico 3.4.1. 
Tabela 2 - Scores de eficiência e média da eficiência

\begin{tabular}{l|c|c|c|c|c}
\hline \multicolumn{1}{c|}{ UF } & $\mathbf{2 0 0 4}$ & $\mathbf{2 0 0 9}$ & $\mathbf{2 0 1 3}$ & $\begin{array}{c}\text { Analise de } \\
\text { Janela }\end{array}$ & \multirow{2}{*}{ Ranking } \\
\cline { 2 - 5 } & Eficiência & Eficiência & Eficiência & $\begin{array}{c}\text { Média da } \\
\text { Eficiência }\end{array}$ & \\
\hline Acre & 1,00 & 1,00 & 1,00 & $100,00 \%$ & 1 \\
Alagoas & 1,00 & 1,00 & 1,00 & $100,00 \%$ & 1 \\
Amapá & 1,00 & 1,00 & 1,00 & $100,00 \%$ & 1 \\
Ceará & 1,00 & 1,00 & 1,00 & $100,00 \%$ & 1 \\
Distrito Federal & 1,00 & 1,00 & 1,00 & $100,00 \%$ & 1 \\
Mato Grosso & 1,00 & 1,00 & 1,00 & $100,00 \%$ & 1 \\
Mato Grosso do Sul & 1,00 & 1,00 & 1,00 & $100,00 \%$ & 1 \\
Minas Gerais & 1,00 & 1,00 & 1,00 & $100,00 \%$ & 1 \\
Paraíba & 1,00 & 1,00 & 1,00 & $100,00 \%$ & 1 \\
Piauí & 1,00 & 1,00 & 1,00 & $100,00 \%$ & 1 \\
Rio Grande do & 1,00 & 1,00 & 1,00 & $100,00 \%$ & 1 \\
Norte & 1,00 & 1,00 & 1,00 & $100,00 \%$ & 1 \\
Rio Grande do Sul & 1,00 & 1,00 & 1,00 & $100,00 \%$ & 1 \\
Rondônia & 1,00 & 1,00 & 1,00 & $100,00 \%$ & 1 \\
Roraima & 1,00 & 1,00 & 1,00 & $100,00 \%$ & 1 \\
Santa Catarina & 1,00 & 1,00 & 1,00 & $100,00 \%$ & 1 \\
Tocantins & 1,00 & 1,00 & 0,81 & $95,22 \%$ & 2 \\
Bahia & 0,81 & 1,00 & 1,00 & $95,20 \%$ & 3 \\
Pará & 1,00 & 0,84 & 1,00 & $91,88 \%$ & 4 \\
Maranhão & 1,00 & 0,84 & 1,00 & $91,80 \%$ & 5 \\
Paraná & 0,64 & 1,00 & 1,00 & $91,11 \%$ & 6 \\
Permambuco & 1,00 & 0,80 & 1,00 & $90,10 \%$ & 7 \\
Goiás & 1,00 & 0,71 & 1,00 & $85,32 \%$ & 8 \\
Sergipe & 1,00 & 0,78 & 0,79 & $83,83 \%$ & 9 \\
São Paulo & 0,77 & 0,72 & 1,00 & $80,19 \%$ & 10 \\
Espírito Santo & 0,72 & 0,66 & 0,63 & $66,72 \%$ & 11 \\
Rio de Janeiro & 0,48 & 0,54 & 0,47 & $50,48 \%$ & 11 \\
Amazonas & & & & & \\
\hline
\end{tabular}

Fonte: Elaborado pelos autores.

A Tabela 3 apresenta os resultados dos escores do Índice Composto do modelo de desempate. Pode-se observar que o Índice Composto cumpre seu papel e desempata as DMUs eficientes. Considerando a média dos valores obtidos com a Análise de Janela feita com os Índices Compostos, apenas Santa Catarina é considerada eficiente. O Rio de Janeiro e o Amazonas mantiveram-se nas duas últimas posições.

Tabela 3 - Scores do Índice Composto e média do índice 


\begin{tabular}{|c|c|c|c|c|c|}
\hline \multirow{2}{*}{ UF } & 2004 & 2009 & 2013 & $\begin{array}{c}\text { Analise de } \\
\text { Janela }\end{array}$ & \multirow{2}{*}{ Ranking } \\
\hline & $\begin{array}{c}\text { Índice } \\
\text { Composto }\end{array}$ & $\begin{array}{c}\text { Índice } \\
\text { Composto }\end{array}$ & $\begin{array}{c}\text { Índice } \\
\text { Composto }\end{array}$ & $\begin{array}{l}\text { Média do } \\
\text { Índice }\end{array}$ & \\
\hline Acre & 0,65 & 0,71 & 0,75 & 0,37 & 18 \\
\hline Alagoas & 0,65 & 0,71 & 0,75 & 0,34 & 21 \\
\hline Amapá & 0,66 & 0,71 & 0,75 & 0,73 & 2 \\
\hline Amazonas & 0,26 & 0,29 & 0,35 & 0,18 & 27 \\
\hline Bahia & 0,58 & 0,71 & 0,37 & 0,34 & 23 \\
\hline Ceará & 0,74 & 0,71 & 0,75 & 0,38 & 17 \\
\hline Distrito Federal & 0,65 & 0,71 & 0,75 & 0,67 & 4 \\
\hline Espírito Santo & 0,76 & 0,58 & 0,75 & 0,49 & 13 \\
\hline Goiás & 0,93 & 0,71 & 1,00 & 0,49 & 14 \\
\hline Maranhão & 0,60 & 0,71 & 0,45 & 0,34 & 22 \\
\hline Mato Grosso & 0,76 & 0,69 & 0,47 & 0,50 & 12 \\
\hline Mato Grosso do Sul & 0,90 & 0,83 & 0,84 & 0,57 & 7 \\
\hline Minas Gerais & 0,86 & 0,71 & 0,75 & 0,54 & 8 \\
\hline Pará & 0,70 & 0,86 & 0,51 & 0,51 & 10 \\
\hline Paraíba & 0,79 & 0,83 & 0,94 & 0,47 & 15 \\
\hline Paraná & 0,71 & 0,76 & 0,67 & 0,58 & 6 \\
\hline Pernambuco & 0,47 & 0,71 & 0,89 & 0,33 & 24 \\
\hline Piauí & 0,65 & 0,71 & 0,88 & 0,34 & 20 \\
\hline Rio de Janeiro & 0,60 & 0,51 & 0,53 & 0,27 & 26 \\
\hline $\begin{array}{l}\text { Rio Grande do } \\
\text { Norte }\end{array}$ & 0,73 & 0,82 & 0,94 & 0,45 & 16 \\
\hline Rio Grande do Sul & 0,92 & 0,90 & 0,88 & 0,66 & 5 \\
\hline Rondônia & 0,91 & 0,71 & 0,75 & 0,50 & 11 \\
\hline Roraima & 0,65 & 0,71 & 0,75 & 0,67 & 3 \\
\hline Santa Catarina & 1,00 & 1,00 & 0,76 & 1,00 & 1 \\
\hline São Paulo & 0,88 & 0,25 & 0,30 & 0,36 & 19 \\
\hline Sergipe & 0,74 & 0,47 & 0,59 & 0,28 & 25 \\
\hline Tocantins & 0,83 & 0,86 & 0,88 & 0,52 & 9 \\
\hline
\end{tabular}

Fonte: Elaborado pelos autores.

Pará, Paraná, Goiás, e Espírito Santo, foram os que mais subiram de posição, enquanto Ceará, Acre, Piauí, e Alagoas foram os que mais caíram. Com exceção das DMUs citadas, as demais das 11 últimas posições continuaram entre os últimos 11. Logo, os demais que eram eficientes, continuaram entre os primeiros, embora sejam considerados ineficientes após o desempate.

\section{CONCLUSÕES}

No presente artigo foi proposto um modelo para analisar a eficiência da segurança alimentar utilizando DEA e o método de desempate de Leta et al. (2005) em conjunto com análise de janela para desempatar as DMUs eficientes. 
Foram utilizadas as seguintes variáveis: transferências de recursos para o Programa Bolsa Família (BF) e o PIB per capita (Y) como inputs; pessoas em situação de segurança alimentar (SA) e a expectativa de vida (EV) como outputs; o inverso do percentual de pobres (P), extremamente pobres (EP), desnutrição infantil de crianças (DI), e a taxa de mortalidade infantil (MI) como outputs indesejáveis.

De forma geral, os resultados mostraram que todos possuem outputs proporcionalmente semelhantes, obtendo DMUs empatadas como eficientes. Após o desempate, os resultados apontaram que a única DMU eficiente é Santa Catarina, enquanto Rio de Janeiro e Amazonas se mantiveram nas últimas posições, tendo maiores potenciais de melhora para a segurança alimentar (já que foram ineficientes em todas as situações), caso considerem estados eficientes como seus benchmarkings.

Pode-se sugerir estudos de casos dessas 3 DMUs (Santa Catarina, Rio de Janeiro e Amazonas) como pesquisas futuras para aprofundar a temática da segurança alimentar, verificar as discrepâncias e como realmente melhorar na prática.

\section{Referências}

ANAND, S.; HARRIS, C. 9 .Food and Standard of Living: An Analysis Based on Sri Lankan Data 370. The political Economy of Hunger, p. 297, 1990.

BANKER, R. D.; CHARNES, A.; COOPER, W. W. Some models for estimating technical and scale inefficiencies in data envelopment analysis. Management Science, v. 30, n. 9, p. 1078-1092, 1984.

BICKEL, G. et al. Guide to measuring household food security. US Department of Agriculture, Food and Nutrition Service, Office of Analysis, Nutrition, and Evaluation., 2000.

BRASIL.Conheça o Bolsa Família. Ministério do Desenvolvimento Social e Agrário. Disponível em: https://mds.gov.br/assuntos/bolsa-familia/o-que-e. Acesso em: abr 2017a.

. Escala brasileira de segurança alimentar. Ministério Público Federal, 2004b.

. Lei $\mathbf{n}^{0}$ 10.836, de 09 de janeiro de 2004. Unificação de ações de transferência de renda do Governo Federal. Diário Oficial da União, 2004a.

.Lei $\mathrm{n}^{\circ}$ 11.346, de 15 de setembro de 2006. Cria o Sistema Nacional de Segurança Alimentar e Nutricional (SISAN) com vistas em assegurar o direito humano à alimentação adequada e dá outras 
providências. Diário Oficial da União, 2006.

.Transparência de Recursos para o Bolsa Família. Ministério da Transparência, Fiscalização e Controladoria- Geral da União. 2017b.

CAMIOTO, F. C.; MARIANO, E. B.; REBELATTO, D. A. N. Efficiency in Brazil's industrial sectors in terms of energy and sustainable development. Environmental Science \& Policy, v. 37, p. 50-60, 2014.

CARVALHO, A. T. D. et al. Métodos de análise em programas de segurança alimentar e nutricional: uma experiência no Brasil. Ciênc. saúde coletiva, v. 18, n. 2, p. 309-321, 2013.

CHARNES, A.; COOPER, W. W.; RHODES, E. Measuring the efficiency of decision making units. European Journal of Operational Research, v. 2, n. 6, p. 429-444, 1978.

COELLI, T. J. et al. An Introduction to efficiency and productivity analysis. 2nded. Berlin: Springer, 2005.

COTTA, R. M. M.; MACHADO, J. C. Programa Bolsa Família e segurança alimentar e nutricional no Brasil: revisão crítica da literatura. Rev Panam Salud Publica, v. 33, n. 1, p. 54-60. 2013.

CUSTÓDIO, M. B.; YUBA, T. Y.; CYRILLO, D. C. Política de segurança alimentar e nutricional no Brasil: uma análise da alocação de recursos. Rev Panam Salud Publica, v. 33, n. 2, p.144-50, 2013.

DATASUS. Número de crianças desnutridas até 12 meses de idade. Departamento de Informática do Sistema Único de Saúde 2017a.

. Taxa de mortalidade infantil. Departamento de Informática do Sistema Único de Saúde $2017 b$.

FARRELL, M. J. The measurement of productive efficiency. Journal of the Royal Statistical Society. Series A (General), v. 120, n. 3, p. 253-290, 1957.

FERREIRA VINHAS, A. L. (In) segurança alimentar no Brasil: uma análise das políticas públicas dos governos de Lula. Cuadernos de Geografía-Revista Colombiana de Geografía, n. 19, p. 177 $186,2010$. 
GREENE, W. H. Econometric analysis. Pearson Education India, 2003.

HOFFMANN, R. Brasil, 2013: mais segurança alimentar. Segurança Alimentar e Nutricional, v. 21, n. 2, p. 422-436, 2014.

FUNDO MONETÁRIO INTERNACIONAL. FMI. World Economic Outlook: Too Slow for too long. Fundo Monetário Internacional, 2017.

INSTITUTO DE PESQUISA ECONÔMICA APLICADA. IPEA. Número de pobres. Instituto de Pesquisa em Economia Aplicada. 2017a.

. Número de extremamente pobres. Instituto de Pesquisa em Economia Aplicada. 2017b.

. Expectativa de vida. Instituto de Pesquisa em Economia Aplicada. 2017c.

MARIANO, E. B.; REBELATTO, D. A. D. N. Transformation of wealth produced into quality of life: analysis of the social efficiency of nation-states with the DEA's triple index approach. Journal of the Operational Research Society, v. 65, n. 11, p. 1664-1681, 2014.

MARTINS, A. P. B. et al. Transferência de renda no Brasil e desfechos nutricionais: revisão sistemática. Revista de Saúde Pública, v. 47, n. 6, p. 1159-1171, 2013.

PESQUISA NACIONAL POR AMOSTRA DE DOMICÍlIOS. PNAD. Pesquisa Suplementar de Segurança Alimentar 2004/2009, 2017a.

PESQUISA NACIONAL POR AMOSTRA DE DOMICÍLIOS. PNAD. Pesquisa Suplementar de Segurança Alimentar 2013, $2017 \mathrm{~b}$.

RADIMER, K. L. et al. Understanding hunger and developing indicators to assess it in women and children. Journal of Nutrition Education, v. 24, n. 1, p. 36S-44S, 1992

RAMOS, C. I.; CUERVO, M. R. M. Programa Bolsa Família: a interface entre a atuação profissional e o direito humano a alimentação adequada. Ciência \& Saúde Coletiva, v. 17, n. 8, p. 2159-2168, 2012. 
VILELA, D. L. Utilização do método analise envoltória de dados para avaliação do desempenho econômico de cooperativas de crédito. 2004. 144p. Dissertação (Mestrado em Engenharia de Produção) - Escola de Engenharia de São Carlos, Universidade de São Paulo, São Carlos, 2004. WOLF, M. R.; BARROS FILHO, A. D. A. Estado nutricional dos beneficiários do Programa Bolsa Família no Brasil-uma revisão sistemática. Ciência \& Saúde Coletiva, v.19, n.5, p.1331-1338, 2014. 